\title{
ESTILOS DE LIDERANÇA E AUTOGESTÃO EM EQUIPES ÁGEIS: A LACUNA DO
} COMPORTAMENTO DO LÍDER

Daniel Capaldo Amaral (amaral@sc.usp.br) - Escola de Engenharia de São Carlos, Universidade de São Paulo (USP).

Natália Pereira dos Anjos Silva (natalia.anjos@usp.br) - Escola de Engenharia de São Carlos, Universidade de São Paulo (USP).

\section{RESUMO}

Os métodos ágeis surgiram como resposta para o gerenciamento de projetos do tipo inovador e são cada vez mais comuns na área de tecnologia. Eles dependem de equipes autogeridas e a presença de lideranças para implantação destas equipes é fundamental. Mas, qual é o estilo de liderança recomendado para equipes ágeis autogeridas? Nesta pesquisa apresenta-se a revisão sistemática sobre as recomendações de estilos de liderança para equipes ágeis autogeridas. $\mathrm{O}$ resultado demonstra não haver consenso sobre o estilo de liderança, evidenciando lacunas na compreensão da relação entre estilo de liderança e autogestão em equipes ágeis. Como resultado, demonstra-se que o principal entrave é a compreensão sobre como deve ser o comportamento dos líderes para a introdução da autogestão. Este resultado abre caminhos para novas pesquisas que podem auxiliar na introdução de modelos ágeis de gestão.

Palavras chave: gestão de projetos ágeis, autogestão, estilos de liderança. 


\section{INTRODUÇÃO}

Segundo Hoda, Noble e Marshall (2013), as equipes auto-organizadas foram reconhecidas e estudadas de várias formas, como grupos autônomos em sistemas sociotécnicos, agentes de gestão do conhecimento, facilitadores de teorias organizacionais, e como exemplos de Sistemas Adaptativos Complexos. O conceito de equipes autogeridas foi incorporado em projetos ágeis e ficou conhecido como um dos elementos centrais no Manifesto Ágil de Software em 2001 (AMARAL et al., 2011). De acordo com o Manifesto Ágil, as melhores arquiteturas, requisitos e designs emergem de equipes auto-organizáveis (HIGHSMITH; FOWLER, 2001).

As equipes autogeridas são grupos autônomos ou empoderados, conforme Dyba, Dingsøyr e Moe (2014), com autoridade e responsabilidade para estabelecer o funcionamento próprio (SOLANSKY, 2008). A pesquisa de Hoegl e Parboteeah (2006) mostra que, no contexto de projetos inovadores, os altos níveis de influência igualitária dos membros da equipe sobre as decisões do projeto são benéficos para facilitar os processos colaborativos para os quais as equipes de inovação multifuncionais são criadas.

A transição para equipes autogerenciadas é um dos maiores desafios ao introduzir os métodos ágeis pois, de acordo com Moe, Dingsøyr e Dybå (2010), não é suficiente agrupar os indivíduos e denominar o time como "autogerenciado" e esperar que entendam automaticamente como funciona o trabalho e a sua coordenação em uma equipe ágil. A transição do trabalho individual para equipes autogerenciadas requer uma reorientação, não apenas dos desenvolvedores, mas também da gerência (MOE; DINGSØYR; DYBÅ, 2010).

Highsmith (2009) menciona que as equipes autogeridas não são equipes sem liderança, pois direção, alinhamento e comprometimento ainda são essenciais como resultados da liderança (DRATH et al., 2008). Assim, os líderes de equipes ágeis facilitam a autoorganização da equipe, removendo os obstáculos do caminho do time (SRIVASTAVA; JAIN, 2017) e atuam como mentores (HODA; NOBLE; MARSHALL, 2010), responsáveis por desenvolver os membros da equipe (MARNEWICK; MARNEWICK, 2020) fornecendo treinamento e recursos necessários para a realização das tarefas do projeto (SRIVASTAVA; JAIN, 2017), além de contribuir para estimular a autonomia dos membros da equipe (MARNEWICK; MARNEWICK, 2020).

Há um conjunto de pesquisas realizadas sobre o perfil de liderança em equipes ágeis. Autores como Bäcklander (2019), Marnewick e Marnewick (2020) e Parker, Holesgrove e Pathak (2015) apresentam propostas de estilos de liderança recomendados para atuar em 
equipes ágeis autogeridas. Mas, haveria algum estilo específico para equipes ágeis? Quais são os estilos mais recomendados? O presente estudo revisa os estilos de liderança identificados na literatura sobre autogestão em equipes ágeis de projeto.

\section{REVISÃO TEÓRICA}

\subsection{Liderança em equipes ágeis autogeridas}

Segundo Northouse (2013), ao longo dos anos a liderança foi definida e conceituada de várias maneiras. Northouse (2013, p. 5) define liderança como: "um processo pelo qual um indivíduo influencia um grupo de indivíduos para alcançar um objetivo comum”. No caso de equipes ágeis, deve-se ter como pressuposto que a liderança pode ser assumida por todos os membros da equipe e, segundo Solansky (2008), não possuem um líder formal designado.

Segundo Moe, Dingsøyr e Kvangardsnes (2009), para que a equipe autogerida tenha autonomia interna, é necessário compartilhar a liderança e a autoridade de decisão entre os membros do time, ao invés de uma estrutura de decisão centralizada ou descentralizada (HOEGL; PARBOTEEAH, 2006). As equipes podem delegar autoridade sobre certas decisões a indivíduos ou subgrupos dentro da equipe segundo a competência ou experiência na especialidade de cada membro da equipe, então nem sempre há envolvimento igual de todos os membros da equipe sobre as decisões do projeto (HOEGL; PARBOTEEAH, 2006).

Observando recomendações de pesquisas sobre o conjunto de práticas orientadoras para o estabelecimento das lideranças em equipes ágeis, notam-se diferentes perspectivas. Por exemplo, Strode (2015) afirma que o líder da equipe ágil autogerida deve sincronizar as contribuições individuais dos membros da equipe e também que, essa liderança deve ser compartilhada com os membros da equipe; Hoda, Noble e Marshall (2010) apresentam papéis adotados pelas equipes ágeis de modo a facilitar a auto-organização do time; e Marnewick e Marnewick (2020) destacam que a liderança Servidora é a melhor maneira de gerenciar equipes em um ambiente ágil. Uma forma de auxiliar a transição para a autogestão seria compreender qual o estilo de liderança a ser desenvolvido nas equipes ágeis. A próxima seção explora especificamente os estilos de liderança em equipes ágeis autogeridas. 


\subsection{Estilos de liderança em equipes ágeis autogeridas}

Armstrong (2012) afirma que o estilo de liderança é a abordagem que os gerentes usam para exercer a liderança quando se relacionam com os membros da equipe e, segundo Northouse (2013), consiste no padrão de comportamento de uma pessoa que tenta influenciar os outros. A abordagem de estilo é diferente das abordagens de traços e habilidades para liderança, pois a abordagem de estilo se concentra no que os líderes fazem e como agem, e não em quem são os líderes, enfatizando o comportamento do líder (NORTHOUSE, 2013). Northouse (2013), afirma que o objetivo principal da abordagem do estilo é explicar como os líderes combinam os dois tipos de comportamento, de tarefa e relacionamento, para influenciar os subordinados em seus esforços para atingir uma meta.

Os estilos de liderança devem ser selecionados e adaptados para se adequar às organizações, situações, grupos e indivíduos (AMANCHUKWU; STANLEY; OLOLUBE, 2015). Segundo Akparep, Jengre e Mogre (2019), o estilo de liderança de uma organização possui forte influência sobre o desempenho, satisfação e comprometimento dos funcionários.

Há diversos fatores que podem ajudar a determinar qual estilo de liderança é mais eficaz e/ou quando recorrer a um estilo diferente ou a uma combinação de estilos, como: tamanho da organização, grau de interação/comunicação, personalidade dos membros, coerência de metas e nível de tomada de decisão (IBARA ${ }^{1}, 2010$, p. 74-76 apud AMANCHUKWU; STANLEY; OLOLUBE, 2015 p. 10-11).

De acordo com Armstrong (2012), existem diversos estilos de liderança e nenhum deles é necessariamente melhor do que outro, e os líderes eficazes são capazes de flexionar seu estilo para atender às demandas da situação. Os estilos de liderança apresentados na literatura incluem por exemplo, as lideranças Autocrática, Burocrática, Carismática, Democrática ou Participativa, Laissez-faire, Transacional, Transformacional, Servidora, Ideológica, Pragmática, Autêntica, Ética e Espiritual (AMANCHUKWU; STANLEY; OLOLUBE, 2015; ANDERSON; SUN, 2015).

No caso das equipes ágeis autogeridas, há estudos que recomendam estilos de liderança para essas equipes, como pode ser visto em Bäcklander (2019), Marnewick e Marnewick (2020) e Parker, Holesgrove e Pathak (2015), por exemplo. Entretanto, não são todos os autores que nomeiam como estilos de liderança o que se refere ao conjunto de comportamentos adotados pelos líderes para influenciar os subordinados a atingir uma meta,

\footnotetext{
1 IBARA, E. C. Perspectives in Educational Administration. Port Harcourt: Rodi Printing and Publishing, 2010 .
} 
como nos estudos de Hoda, Noble e Marshall (2010), Srivastava e Jain (2017) e Barke e Prechelt (2019), por exemplo.

Um primeiro exemplo de estilo de liderança é a Servidora. Greenleaf (1977) afirma que os líderes Servidores constroem confiança servindo ao próximo. Nesse sentido, o ato de servir se estende além dos desejos do ego e constrói um clima de trabalho que gera sentimentos de empoderamento do funcionário e estimula a autoconfiança nos membros da equipe, conforme Liden et al. (2008), o que beneficia a construção de equipes autogeridas.

O estilo de liderança Capacitadora é descrito nos estudos de Bäcklander (2019), UhlBien e Arena (2018) e Uhl-Bien, Marion e McKelvey (2007). Eles indicam que a liderança Capacitadora permite o equilíbrio entre liberdade e alinhamento, exigidos em contextos altamente dinâmicos como aqueles que utilizam a abordagem ágil. Uhl-Bien, Marion e McKelvey (2007) ressaltam que a liderança Capacitadora estrutura e possibilita as condições necessárias para que o CAS (Sistema Adaptativo Complexo) seja capaz de abordar de forma otimizada a resolução criativa de problemas, adaptabilidade e aprendizagem na equipe.

Theobald et al. (2020) consideram a existência do perfil de líderes Ágeis. Segundo estes autores, funcionam como agentes de mudança para impulsionar a mudança cultural na organização em direção à abordagem ágil. Ao seguir práticas flexíveis e ágeis, o gerente se torna um líder adaptativo, definindo a direção, estabelecendo os princípios orientadores e incentivando o feedback, a adaptação e a colaboração constantes (PARKER; HOLESGROVE; PATHAK, 2015).

Há ainda os líderes Visionários que, de acordo com Gutierrez et al. (2019), são aqueles que incentivam os membros de sua equipe a revelar o que há de melhor em si mesmos. Os líderes Coaches buscam conectar os objetivos pessoais com os valores da organização (GUTIERREZ et al., 2019). A liderança Afiliativa, conforme Gutierrez et al. (2019), foca em desenvolver conexões emocionais e harmonia ao resolver conflitos. Segundo Gutierrez et al. (2019), os líderes Democráticos promovem a colaboração entre os membros da equipe.

Em suma, os estilos de liderança correspondem a um perfil específico de comportamentos ou atividades que os líderes adotam. Os estilos de liderança identificados para equipes ágeis são: Afiliativa, Ágil, Capacitadora, Coach, Democrática, Servidora e Visionária. As definições de cada autor variam e, portanto, é possível concluir que não há um consenso sobre o estilo mais recomendado para a aplicação da abordagem ágil de gestão, em rumo da autogestão. Uma alternativa é identificar nesses artigos os estilos de liderança 
associados aos comportamentos de liderança citados. Na próxima seção apresenta-se um método com este objetivo e fundamentado na lente teórica dos estilos de liderança segundo Northouse (2013).

\section{METODOLOGIA}

O problema que se busca solucionar nessa pesquisa é: "Quais são os estilos de liderança recomendados para equipes ágeis autogeridas?”. O estudo teve como objetivo coletar todos os comportamentos de liderança em equipes ágeis, relacionando-os com os estilos citados, de forma a compreender qual estilo é recomendado de forma mais precisa. Trata-se de pesquisa qualitativa e descritiva segundo Turrioni e Melo (2012).

Existe um corpo teórico significativo sobre liderança nas áreas de psicologia e organização do trabalho. Este estudo apresenta uma Revisão Bibliográfica Sistemática (RBS) específica sobre as pesquisas de liderança no campo do gerenciamento ágil de projetos e autogestão. Segundo Booth, Sutton e Papaioannou (2016), revisar a literatura de forma sistemática oferece clareza e confiança na pesquisa ao demonstrar o rigor de seus métodos, permite a replicação da pesquisa a partir da transparência dos procedimentos descritos e, como neste caso, pode ajudar a sistematizar o conhecimento sobre o tema.

As etapas da RBS foram adaptadas da RBS Roadmap, de Conforto, Amaral e Silva (2011), buscando analisar o conteúdo dos artigos que abordam liderança em contextos de equipes ágeis autogeridas. A princípio foram selecionados três artigos como fontes primárias, devido à relevância nesta pesquisa: Bäcklander (2019), Moe, Dingsøyr e Dybå (2010) e Marnewick e Marnewick (2020). Para iniciar as buscas da RBS, foi escolhida a base de dados Scopus®. Segundo Elsevier (2021), Scopus® é o maior banco de dados de resumos e citações da literatura com revisão por pares e contempla todas as áreas do estudo: ciências sociais e humanas e tecnologias (ELSEVIER, 2021).

Na RBS dessa pesquisa foram considerados três filtros segundo Conforto, Amaral e Silva (2011): leitura do título, resumo e palavras-chave dos artigos encontrados; leitura da introdução e conclusão dos artigos selecionados no filtro 1; leitura por completo dos artigos selecionados.

Para avaliar quais artigos do resultado de pesquisa seriam aprovados no final da RBS, foram aplicados os critérios de inclusão em cada filtro, definindo o conteúdo que os artigos precisam abordar para serem incluídos na pesquisa. Os critérios de inclusão foram: 1) apresentar revisão bibliográfica sobre liderança em equipes ágeis autogeridas; 2) apresentar 
liderança em equipes ágeis autogeridas como tema principal do texto. Assim, para a inclusão dos artigos na pesquisa, foi necessário atender aos três critérios de inclusão.

Na RBS foi considerado o horizonte de tempo entre 2001-2020, considerando como marco o surgimento do manifesto ágil, identificado na revisão histórica de Amaral et al. (2011). As informações utilizadas no procedimento de busca da RBS são apresentadas no Quadro 1.

Quadro 1 - Informações do procedimento de busca da RBS.

\begin{tabular}{|c|c|}
\hline Base de dados & Scopus $®$ \\
\hline $\begin{array}{l}\text { Período destinado } \\
\text { às buscas }\end{array}$ & $15 / 09 / 2020-18 / 10 / 2020$ \\
\hline $\begin{array}{l}\text { Tempo estipulado } \\
\text { nas buscas }\end{array}$ & 2001-2020 \\
\hline $\begin{array}{l}\text { Strings de busca: } \\
\text { agilidade, } \\
\text { liderança e } \\
\text { autogestão }\end{array}$ & $\begin{array}{l}\text { (TITLE-ABS-KEY ( leader* OR "agile-leader*" OR "agile leader*" OR } \\
\text { "servant leader*" OR "enabling leader*" OR "agile coach*" OR } \\
\text { "empowering leader*" ) ) AND ( ALL ( "self-manag* team*" OR "self- } \\
\text { organiz* team*" OR "self-direct* team*" OR "empower* team*" ) ) AND ( } \\
\text { TITLE-ABS-KEY ( ( "agile" OR "agility" ) AND NOT ( lean OR "agile } \\
\text { manufacturing" ) ) ) }\end{array}$ \\
\hline $\begin{array}{l}\text { Exclusão de áreas } \\
\text { de estudo }\end{array}$ & $\begin{array}{l}\text { medicine OR physics and astronomy OR health professions OR earth and } \\
\text { planetary sciences OR biochemistry, genetics and molecular biology OR } \\
\text { chemical engineering OR chemistry OR nursing OR neuroscience OR } \\
\text { pharmacology, toxicology and pharmaceutics OR immunology and } \\
\text { microbiology OR veterinary OR dentistry }\end{array}$ \\
\hline $\begin{array}{l}\text { Exclusão de tipo } \\
\text { de documento }\end{array}$ & book OR book chapter \\
\hline
\end{tabular}

Fonte: Dados da pesquisa (2020).

As buscas na base de dados do Scopus ${ }^{\circledR}$ apresentaram 39 artigos com os conceitos de agilidade, liderança e autogestão. O procedimento iterativo da RBS, incluindo a quantidade de artigos em cada filtro, estão representados na Figura 1. 
Figura 1 - Procedimento iterativo da revisão bibliográfica sistemática.

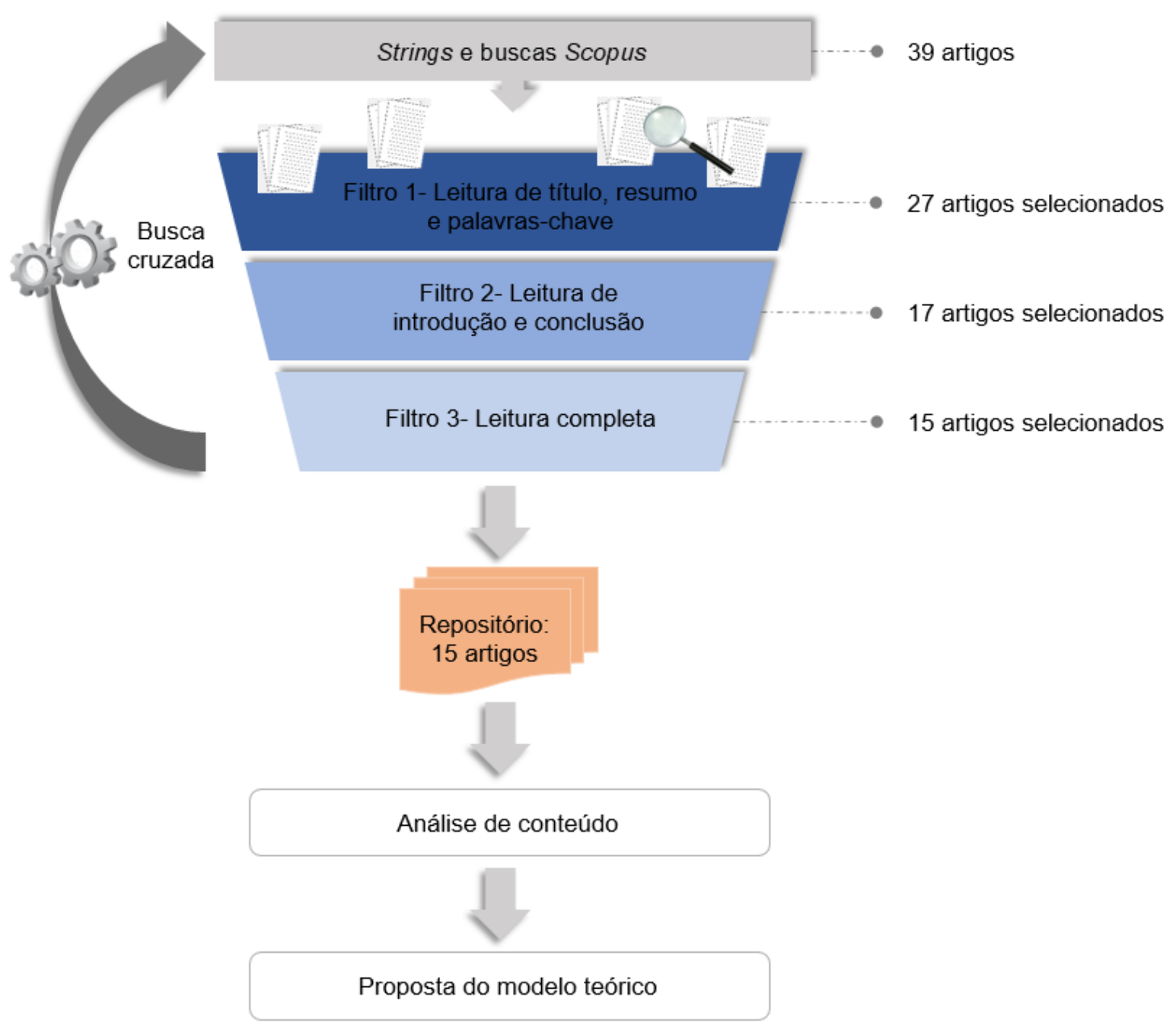

Fonte: Adaptado de Conforto, Amaral e Silva (2011).

Análise de conteúdo foi a técnica utilizada para identificar comportamentos e estilos de liderança recomendados para equipes ágeis nos artigos. A unidade de registro temática definida foi comportamento de liderança por ser comum a todos os textos. A unidade de contexto é a equipe ágil autogerida. A análise de conteúdo iniciou-se a partir da identificação dos comportamentos de liderança citados nos artigos. Nesta etapa foram agrupados os comportamentos similares com registro de frequência de citações. A segunda etapa envolveu a identificação dos estilos de liderança atribuídos diretamente pelos autores para cada comportamento. Restaram comportamentos sem estilos identificados (rotulados como não identificados na planilha). Todos os dados foram analisados e sintetizados em uma planilha pela pesquisadora, com a ajuda de um especialista. 


\section{RESULTADOS E DISCUSSÃO}

Foram encontradas sete categorias para estilo de liderança: Servidora, Ágil, Capacitadora, Visionária, Democrática, Coach e Afiliativa. A Tabela 1 descreve a quantidade de comportamentos associados a cada estilo. Foram registrados 182 comportamentos durante a coleta. Deve-se notar que a maioria dos comportamentos não está associada a um estilo.

Tabela 1 - Frequência de estilos de liderança recomendados para a equipe de projetos ágil autogerida

Fonte: Dados da pesquisa (2020).

\begin{tabular}{l|c}
\hline \multicolumn{1}{c}{ Estilos de liderança } & Frequência \\
\hline Não identificado & 151 \\
Liderança Servidora & 14 \\
Liderança Ágil & 7 \\
Liderança Capacitadora & 6 \\
Liderança Visionária & 1 \\
Liderança Democrática & 1 \\
Liderança Coach & 1 \\
Liderança Afiliativa & 1 \\
\hline
\end{tabular}

É evidente pela Tabela 1 que a maioria dos artigos que citam comportamentos de liderança para equipes ágeis autogeridas não apresentam o estilo de liderança associado ao comportamento, que são os denominados "não identificados" na Tabela 1.

A liderança Servidora é citada com maior frequência para a autogestão em equipes ágeis. A liderança Servidora, segundo Liden et al. (2008), atua de forma a facilitar as atividades dos membros da equipe de projeto, se dedica ao desenvolvimento dos membros do time fornecendo apoio e orientação, o que estimula a autoconfiança da equipe e contribui para promover a autogestão em equipes ágeis.

Os estilos de liderança Ágil e Capacitadora a sucedem na Tabela 1. O estilo de liderança Ágil, proposto por Theobald et al. (2020), atua como agente de mudança para impulsionar a transição cultural na organização em direção à abordagem ágil, orientando e incentivando os membros do time. É uma proposta nova e que descreve ações muito próximas do papel de Scrum Master ou Agilista. A liderança Capacitadora, conforme Uhl-bien, Marion e Mckelvey (2007) possibilita a resolução criativa de problemas, a adaptabilidade e aprendizagem na equipe, destacando a importância desses estilos de liderança para as equipes ágeis. Nota-se que apesar da relevância desses dois estilos de liderança em equipes ágeis autogeridas, os rótulos de liderança Capacitadora e Ágil foram citados por apenas dois textos dentre os artigos selecionados na RBS, sendo um texto para cada um desses estilos de 
liderança. As lideranças Visionária, Democrática, Coach e Afiliativa seguem com menor frequência de citação para equipes ágeis autogeridas.

É notável que a maioria dos comportamentos de liderança citados para equipes ágeis não apresentaram os respectivos estilos de liderança. Esse resultado é interessante pois indica que pode haver comportamentos do líder que seriam específicos para o contexto de equipes ágeis de projetos. Além disso, a recomendação de sete estilos de lideranças distintos evidencia que ainda não há um consenso entre os estilos de liderança recomendados para equipes ágeis

\section{CONCLUSÃO}

Os principais resultados incluem a identificação de sete estilos de liderança para equipes ágeis autogeridas, Servidora, Ágil, Capacitadora, Visionária, Democrática, Coach e Afiliativa, e a comprovação que os estudos que abordaram lideranças e equipes ágeis não conseguiram caracterizar ainda qual o melhor estilo. Segundo o levantamento, eles focam em papéis de liderança e seus comportamentos.

Apesar da não existência de um consenso e da maioria dos estudos não identificar estilos de liderança recomendados para equipes ágeis, a liderança Servidora foi a mais recomendada e descobriu-se um estilo de liderança específico com o nome Ágil. Portanto, há aqui uma lacuna e faz-se necessário um estudo aprofundado dos comportamentos dos integrantes de líderes em equipes ágeis tal que se possa identificar uma recomendação de estilo que auxilie os profissionais de gestão de projetos e de recursos humanos no desenvolvimento e aquisição de recursos humanos para compor este tipo de equipe.

Uma limitação para o presente estudo envolve a análise restrita de dados, já que foram considerados e analisados somente as informações advindas da literatura, demonstrando a necessidade de pesquisas de campo sobre estes estilos de liderança identificados em equipes ágeis.

Como pesquisa futura, portanto, propõe-se identificar quais são os estilos de liderança que têm sido adotados em equipes ágeis e comparar com os resultados da RBS deste estudo ou com os construtos e instrumentos clássicos da área de liderança, que apontam os comportamentos de um líder. Isso permitiria averiguar se os estilos de liderança identificados na literatura são realmente utilizados nas organizações que passaram pela transição para a abordagem ágil, permitindo também identificar novos estilos de liderança ainda não citados na literatura e analisar se, na prática, faz sentido a criação de um novo estilo de liderança 
denominado ágil. Esse caminho é necessário para um diagnóstico de liderança útil na transição em direção às equipes ágeis autogeridas.

\section{REFERÊNCIAS}

AKPAREP, J. Y.; JENGRE, E.; MOGRE, A. A. The Influence of Leadership Style on Organizational Performance at TumaKavi Development Association, Tamale, Northern Region of Ghana. Open Journal of Leadership, v. 8 , n. 1, p. 1-22, 2019.

AMANCHUKWU, R. N.; STANLEY, G. J.; OLOLUBE, N. P. A Review of Leadership Theories, Principles and Styles and Their Relevance to Educational Management. Management, v. 5, n. 1, p. 6-14, 2015.

AMARAL, D. C. et al. Gerenciamento ágil de projetos: aplicação em produtos inovadores. São Paulo: Saraiva, 2011.

ANDERSON, M. H.; SUN, P. Y. T. Reviewing Leadership Styles: Overlaps and the Need for a New 'Full-Range' Theory. International Journal of Management Reviews, p. 1-21, 2015.

ARMSTRONG, M. Armstrong's Handbook of Management and Leadership: Developing effective people skills for better leadership and management. 3. ed. Kogan Page, 2012.

BACKLANDER, G. Doing complexity leadership theory: How agile coaches at Spotify practise enabling leadership. Creativity and innovation management, v. 28, n.1, p. 42-60, 2019.

BARKE, H.; PRECHELT, L. Role clarity deficiencies can wreck agile teams. PeerJ Comput, Berlin, p. 1-20, 2019.

BOOTH, A.; SUTTON, A.; PAPAIOANNOU, D. Systematic approaches to a successful literature review. 3. ed. SAGE, 2016.

CONFORTO, E. C.; AMARAL, D. C.; SILVA, S. L. Roteiro para revisão bibliográfica sistemática: aplicação no desenvolvimento de produtos e gerenciamento de projetos. $\mathbf{8}^{\mathbf{0}}$ Congresso Brasileiro de Gestão de Desenvolvimento de Produto, Porto Alegre, p. 1-12, 2011.

DRATH, W. H.; MCCAULEY, C. D.; PALUS, C. J.; VELSOR, E. V.; O'CONNOR, P. M. G.; MCGUIRE, J. B. Direction, alignment, commitment: Toward a more integrative ontology of leadership. The Leadership Quarterly, v. 19, n. 6, p. 635-653, 2008.

DYBÅ, T.; DINGS $\varnothing Y R$, T.; MOE, N. B. Agile Project Management. In: RUHE, G.; WOHLIN, C. Software Project Management in a Changing World. Berlin: Springer, 2014. 11, p. 277-300.

ELSEVIER. Scopus - O maior banco de dados da literatura revisada por pares. 2021. Disponível em: <https://www.elsevier.com/pt-br/solutions/scopus >. Acesso em: 13 abr. 2021.

GREENLEAF, R. K. Servant Leadership: A Journey Into the Nature of Legitimate Power and Greatness. New York: Paulist Press, 1977.

GUTIÉRREZ, G.; GARZÁS, J.; LENA, M. T. G.; MOGUERZA, J. M. Self-Managing: An Empirical Study of the Practice in Agile Teams. IEEE SOFTWARE, v. 36, n. 1, p. 23-27, 2019. 
HIGHSMITH, J. Agile Project Management: Creating Innovative Products. 2. ed. AddisonWesley Professional, 2009.

HIGHSMITH, J.; FOWLER, M. The Agile Manifesto. Software Development, 2001.

HODA, R.; NOBLE, J.; MARSHALL, S. Organizing Self-Organizing Teams. IEEE International Conference on Software Engineering, Cape Town, v. 1, p. 285-294, 2010.

HODA, R.; NOBLE, J.; MARSHALL, S. Self-Organizing Roles on Agile Software Development Teams. IEEE Transactions on Software Engineering, v. 39, n. 3, p. 422-444, 2013.

HOEGL, M.; PARBOTEEAH, P. Autonomy and teamwork in innovative projects. Human Resource Management, v. 45, n. 1, p. 67-79, 2006.

LIDEN, R. C.; WAYNE, S. J.; ZHAO, H.; HENDERSON, D. Servant leadership: development of a multidimensional measure and multi-level assessment. The Leadership Quarterly, v. 19, n. 2, p. 161-177, 2008.

MARNEWICK, A. L.; MARNEWICK, C. The Ability of Project Managers to Implement Industry 4.0-Related Projects. IEEE ACCESS, v. 8, p. 314-324, 2020.

MOE, N. B.; DINGSØYR, T.; DYBÅ, T. Teamwork model for understanding an agile team: A case study of a Scrum project. Information and Software Technology, v. 52, n. 5, p. 480491, 2010.

MOE, N. B.; DINGS $\varnothing Y R$, T.; KVANGARDSNES, Ø. Understanding Shared Leadership in Agile Development: A Case Study. IEEE Xplore, p. 1-10, 2009.

NORTHOUSE, P. G. Leadership: Theory and Practice. 6. ed. Sage Publications, 2013.

PARKER, D. W.; HOLESGROVE, M.; PATHAK, R. Improving productivity with selforganised teams and agile leadership. International Journal of Productivity and Performance Management, v. 64, n. 1, p. 112-128, 2015.

SOLANSKY, S. T. Leadership Style and Team Processes in Self-Managed Teams. Journal of Leadership \& Organizational Studies, v. 14, n. 4, p. 332-341. 2008.

SRIVASTAVA, P.; JAIN, S. A leadership framework for distributed self-organized scrum teams. Team Performance Management, v. 23, n. 5/6, p. 293-314. 2017.

STRODE, D. Applying Adapted Big Five Teamwork Theory to Agile Software Development. Proceedings of the 26th Australasian Conference on Information Systems, Adelaide, 2015.

THEOBALD, S.; PRENNER, N.; KRIEG, A.; SCHNEIDER, K. Agile Leadership and Agile Management on Organizational Level - A Systematic Literature Review. In: MORISIO, M.; TORCHIANO, M.; JEDLITSCHKA, A. Product-Focused Software Process Improvement. Springer, 2020. p. 20-36.

TURRIONI, J. B.; MELLO, C. H. P. Metodologia de pesquisa em engenharia de produção: estratégias, métodos e técnicas para condução de pesquisas quantitativas e qualitativas. 2012. Universidade Federal de Itajubá, Itajubá, 2012.

UHL-BIEN, M.; ARENA, M. Leadership for organizational adaptability: A theoretical synthesis and integrative framework. The Leadership Quarterly, v. 29, n. 1, p. 89-104, 2018. 
UHL-BIEN, M.; MARION, R.; MCKELVEY, B. Complexity Leadership Theory: Shifting leadership from the industrial age to the knowledge era. The Leadership Quarterly, v. 18, n. 4, p. 298-318, 2007. 